\title{
Signal-mediated interactions between Pseudomonas aeruginosa and Candida albicans
}

Correspondence

John P. Morrissey

j.morrissey@ucc.ie

Received 18 October 2007

Accepted 29 January 2008

\author{
Gordon McAlester, ${ }^{1}$ Fergal O'Gara ${ }^{1,2}$ and John P. Morrissey ${ }^{1}$ \\ ${ }^{1}$ Department of Microbiology, University College Cork, Ireland \\ ${ }^{2}$ BIOMERIT Research Centre, Biosciences Institute, University College Cork, Ireland
}

\begin{abstract}
Pseudomonas aeruginosa causes infections in a wide variety of hosts and is the leading cause of mortality in cystic fibrosis (CF) patients. Although most clinical isolates of $P$. aeruginosa share common virulence determinants, it is known that strains evolve and change phenotypically during $\mathrm{CF}$ lung infections. These changes can include alterations in the levels of $\mathrm{N}$-acyl homoserine lactones (HSLs), which are secreted signal molecules. In the CF lung, fungi, especially Candida albicans and Aspergillus fumigatus, may coexist with $P$. aeruginosa but the implications for disease are not known. Recent studies have established that signalling can occur between $P$. aeruginosa and C. albicans, with the bacterial molecule 3-oxo-C12HSL affecting Candida morphology, and the fungal metabolite farnesol reducing levels of the Pseudomonas quinolone signal and pyocyanin in Pseudomonas. Whether these interactions are common and typical in clinical strains of $P$. aeruginosa was addressed using CF isolates that produced varied levels of HSLs. It was found that, whereas some clinical $P$. aeruginosa strains affected C. albicans morphology, others did not. This correlated closely with the amounts of 3-oxo-C12HSL produced by the isolates. Furthermore, it was established that signalling is bidirectional and that the $C$. albicans molecule farnesol inhibits swarming motility in $P$. aeruginosa CF strains. This work demonstrates that clinical isolates of these opportunistic pathogens can interact in strain-specific ways via secreted signals and illustrates the importance of studying these interactions to fully understand the microbial contribution to disease in polymicrobial infections.
\end{abstract}

\section{INTRODUCTION}

Pseudomonas aeruginosa is a ubiquitous Gram-negative bacterium that is found in a myriad of environments. As well as being an opportunistic human pathogen, it is also capable of causing disease in eukaryotes such as plants, mammals, insects and nematodes (Diggle et al., 2003; Giamarellou, 2000; Rahme et al., 1995, 2000; Tan \& Ausubel, 2000; Whitchurch et al., 2005). It is also the principal cause of mortality in cystic fibrosis (CF) populations (Diggle et al., 2003; Drenkard \& Ausubel, 2002; Govan \& Deretic, 1996; Smith et al., 1996). P. aeruginosa communicates using chemical signals to sense cell density and alter gene expression and virulence factors in a process known as quorum sensing (QS) (Heurlier et al., 2006; Schuster \& Greenberg, 2006; Shiner et al., 2005; Smith \& Iglewski, 2003; Williams, 2002). The major cellcell signalling molecules in $P$. aeruginosa are the $\mathrm{N}$-acyl homoserine lactones (HSLs) 3-oxo-C12HSL and C4HSL, and the quinolone 2-heptyl-3-hydroxy-4-quinolone (Pseudomonas quinolone signal; PQS). QS regulates many

Abbreviations: $\mathrm{CF}$, cystic fibrosis; HSL, N-acyl homoserine lactone; PQS, Pseudomonas quinolone signal; PVC, polyvinyl chloride; OS, quorum sensing. virulence features including the formation of biofilms and production of factors such as hydrogen cyanide, pyocyanin, proteases and rhamnolipids (Calfee et al., 2005; Cugini et al., 2007; Diggle et al., 2003, 2006, 2007; McGrath et al., 2004; Wade et al., 2005).

Together with $P$. aeruginosa, the dimorphic yeast Candida albicans is one of the most commonly isolated microorganisms from CF patient sputum (Bakare et al., 2003; Bauernfeind et al., 1987; Hughes \& Kim, 1973). Although C. albicans is a common non-pathogenic commensal of the skin and mucosal flora of healthy individuals, in compromised individuals it is able to initiate invasive growth that may result in serious disease and death (Hube, 2006; Naglik et al., 2004; Pfaller \& Diekema, 2007). C. albicans can exist as yeast or filamentous forms and, in response to certain cues, including temperature and host factors, it switches from yeast to hyphal (filamentous) growth. Interestingly, both morphological forms are important for virulence and thus the ability to morphologically transform is an important virulence trait (Calderone \& Fonzi, 2001; Gow, 1997; Gow et al., 2002; Liu, 2002; Mitchell, 1998; Whiteway \& Oberholzer, 2004). Like P. aeruginosa, C. albicans employs secreted signals to regulate gene expression and virulence. Actively growing yeast cells 
secrete farnesol, a 12-carbon sesquiterpene, which acts as a virulence factor and a repressor of the switch from yeast to hyphal growth in C. albicans (Enjalbert \& Whiteway, 2005; Hogan, 2006a, b; Hornby et al., 2001; Navarathna et al., 2007a, b; Nickerson et al., 2006).

$P$. aeruginosa and C. albicans are frequently co-isolated from CF patient sputum and a variety of in vitro and in vivo studies have established that these two species can interact. In vitro and in vivo, there is evidence to suggest that $P$. aeruginosa may inhibit $C$. albicans growth in the host (Bauernfeind et al., 1987; Burns et al., 1999; Gupta et al., 2005; Kaleli et al., 2007; Kerr, 1994a, b). Furthermore, several in vitro studies using well-characterized laboratory strains have identified more complex interactions between these human pathogens. Certain strains of $P$. aeruginosa are cytotoxic to the filamentous form of $C$. albicans but are unable to attach to or kill $C$. albicans yeast cells (Hogan \& Kolter, 2002). In addition, it has been reported that 3-oxo-C12HSL and structurally related molecules can inhibit and even reverse the switch from yeast to hyphal growth in C. albicans (Hogan et al., 2004). That study also showed that a mutant defective in producing 3-oxo-C12HSL lost the ability to influence $C$. albicans morphology (Hogan et al., 2004). Interestingly, recent research has shown that these signalling interactions between $P$. aeruginosa and $C$. albicans may in fact be bidirectional. It was found that addition of farnesol leads to decreased PQS production in $P$. aeruginosa and that this decrease leads to a reduction in the PQS-regulated virulence factor pyocyanin (Cugini et al., 2007). Those studies open up an intriguing scenario whereby secreted factors from $P$. aeruginosa and $C$. albicans have mutually antagonistic effects on important virulence traits.

It is known, however, that strains of $P$. aeruginosa isolated from CF patients show significant phenotypic and genotypic variation (Finnan et al., 2004; Martin et al., 1995; Oliver et al., 2000; Wolfgang et al., 2003). These variations in
$P$. aeruginosa $\mathrm{CF}$ strains are likely to alter how $P$. aeruginosa interacts with the host and other organisms including $C$. albicans. In this study, we took advantage of a set of seven characterized $P$. aeruginosa CF clinical isolates to investigate possible interaction between bacterial isolates and the yeast C. albicans. We addressed specifically the question of whether secreted factors from clinical isolates would affect C. albicans growth or morphology. Using cell-free supernatants we were able to focus on the role of HSL molecules in mediating these effects. In addition, we examined the effect of the yeast signal, farnesol, on these bacterial isolates.

\section{METHODS}

Strains, culture conditions and compounds. All strains used in this study (apart from the $\mathrm{HSL}^{-}$mutant) were clinical isolates and are listed in Table 1. All strains were characterized genetically previously and were shown to be genotypically distinct (Adams et al., 1998; Finnan et al., 2004). P. aeruginosa PAO1 and CF strains were routinely cultured at $37{ }^{\circ} \mathrm{C}$ in Luria-Bertani (LB) broth or on LB plates. Antibiotics for selection of the $P$. aeruginosa mutant strain were used at the following concentrations: $100 \mu \mathrm{g}$ gentamicin $\mathrm{ml}^{-1}$ and $200 \mu \mathrm{g}$ tetracycline $\mathrm{ml}^{-1}$. C. albicans was grown in non-filamentinducing medium (YNB) and filament-inducing medium (YNBNP) as described by Hogan et al. (2004). Farnesol (trans, trans-farnesol) was purchased from Fluka and diluted in methanol as required. Purified C12HSL was purchased from Sigma and diluted in acetonitrile as required.

Swarming motility assay. Swarming was analysed on agar plates containing $0.5 \%$ Eiken agar and $8 \mathrm{~g} \mathrm{l}^{-1}$ Eiken nutrient broth (Eiken Chemical Co.) supplemented with $5 \mathrm{~g}$ glucose $\mathrm{l}^{-1}$. Bacteria from an overnight culture were inoculated onto the centre of plates using a sterile pipette tip and the plates were incubated overnight at $37^{\circ} \mathrm{C}$. A positive result was recorded as growth over the agar surface away from the inoculation point. To determine the effect of farnesol, swarming media were supplemented with $100 \mu \mathrm{M}$ farnesol.

Growth and adhesion assays. $P$. aeruginosa growth curves were performed in $\mathrm{LB}$ medium at $37{ }^{\circ} \mathrm{C}$ using a Bioscreen $\mathrm{C}$ microtiterplate reader. To determine the effect of farnesol, LB media were

Table 1. Strains used in this study

\begin{tabular}{|c|c|c|c|}
\hline Strain & Country of isolation & Year of isolation & Reference \\
\hline \multicolumn{4}{|l|}{ Pseudomonas aeruginosa } \\
\hline \multicolumn{4}{|l|}{ Clinical isolates } \\
\hline CF139 & Ireland & 1994 & Adams et al. (1998) \\
\hline CF198 & Ireland & 1994 & Adams et al. (1998) \\
\hline CF95 & Ireland & 1993 & Adams et al. (1998) \\
\hline CF93 & Ireland & 1993 & Adams et al. (1998) \\
\hline CF144 & Ireland & 1994 & Adams et al. (1998) \\
\hline CF177 & Ireland & 1994 & Adams et al. (1998) \\
\hline CF242 & Ireland & 1994 & Adams et al. (1998) \\
\hline \multicolumn{4}{|l|}{ Controls } \\
\hline PAO1 (WT) & & & Holloway \& Morgan (1986) \\
\hline PAO1 1 lasRI:: Gm $\Delta r h l R I:: \mathrm{Tc}$ & & & Beatson et al. (2002) \\
\hline \multicolumn{4}{|l|}{ Candida albicans } \\
\hline SC5314 & & & Gillum et al. (1984) \\
\hline
\end{tabular}


supplemented with 30,100 or $300 \mu \mathrm{M}$ farnesol. Adhesion on polyvinyl chloride (PVC) was measured after $12 \mathrm{~h}$ static incubation using a crystal violet assay essentially as described previously (Burrowes et al., 2006). In experiments to determine the effects of farnesol, $300 \mu \mathrm{M}$ farnesol was added at each starting inoculation.

Detection of HSLs by TLC. HSL extraction with dichloromethane and detection by TLC was carried out as described previously (Baysse et al., 2005) and by Shaw et al. (1997) using the reporter strains Chromobacterium violaceum CV026, capable of sensing short-chain HSL molecules (C4HSL and C6HSL) through the production of a violet pigment (Ravn et al., 2001), and Agrobacterium tumefaciens NTI, which carries a lacZ transcriptional fusion under the control of an HSL-inducible promoter for long-chain HSLs (3-oxo-C12HSL) (Shaw et al., 1997).

Supernatant preparation and morphology studies. $P$. aeruginosa strains were grown overnight for $16 \mathrm{~h}$ in LB liquid media until an approximate $\mathrm{OD}_{600} 1.2$ was reached. Cells were harvested by centrifugation and the supernatant was passed through a Nalgene vacuum filter unit with a $0.2 \mu \mathrm{M}$ polyethersulfone membrane (Sigma). The sterile supernatant was immediately lyophilized and resuspended at a concentration of $\times 20$ in sterile purified water. The sterile, concentrated supernatant was used immediately or stored for short periods of time at $-80{ }^{\circ} \mathrm{C}$ before use. Analysis of C. albicans morphology in liquid medium was carried out as described by Hogan et al. (2004) with minor adjustments. Briefly, C. albicans was grown as yeast cultures overnight at $30{ }^{\circ} \mathrm{C}$ in YNB medium. Yeast cells were diluted in YNBNP filament-inducing medium to $\mathrm{OD}_{600} 0.05$ and incubated at $37^{\circ} \mathrm{C}$. Upon inoculation from YNB to YNBNP, sterile concentrated supernatant from the $P$. aeruginosa strains was added at a final concentration of $\times 2$ from a $\times 20$ stock. After $6 \mathrm{~h}$ growth, cells were examined microscopically at $\times 400$ magnification and the images were digitally captured.

\section{RESULTS AND DISCUSSION}

\section{Production of HSLs is strain dependent}

In a previous study, we surveyed phenotypic characteristics including motility and production of specific virulence factors in a small collection of $P$. aeruginosa $\mathrm{CF}$ isolates (Finnan et al., 2004). No defining virulence traits were identified but, as HSLs have since been shown to exert effects on C. albicans (Hogan et al., 2004), we tested each of the strains for their ability to produce both long- and short-chain HSLs (Fig. 1). HSL production is known to be growth-phase dependent; therefore, we tested each strain at three stages of growth in liquid medium using a wellestablished bioassay. Although not strictly quantitative, this bioassay facilitates comparisons of relative HSL levels among strains. All strains produced the long-chain molecule 3-oxo-C12HSL (Fig. 1, top panel), with clear variation between strains observed. Only three strains, PAO1, CF144 and CF242, produced significant amounts of the short-chain molecules C4HSL and C6HSL, although trace levels could be detected in all strains. Another minor spot, perhaps C5HSL or 3-oxo-C4HSL, was also visible in these strains (Fig. 1, bottom panel, circled spot). HSL production was growth-phase dependent in all cases, with the highest levels being typically associated with stationary phase cultures. It is clear from this analysis that significant variation in HSL production exists within this small group of clinical isolates, with two strains, CF144 and CF242, resembling the laboratory strain PAO1 and categorized as 'high producers' and the five other clinical strains as 'low producers' of long-chain HSLs, short-chain HSLs, or both. These data are consistent with how $P$. aeruginosa is believed to behave in the CF lung. In this condition, the same lineage of $P$. aeruginosa can persist in the lung for years, even decades, undergoing many evolutionary changes due to selective and adaptive pressures within the host (Nguyen \& Singh, 2006; Smith et al., 2006). As an infection progresses from the acute to chronic phase, virulence factors, including production of HSLs, appear to be counter-selected by the host (Nguyen \& Singh, 2006; Smith et al., 2006). Interestingly, our set of CF isolates showed a large degree of variation in their production of
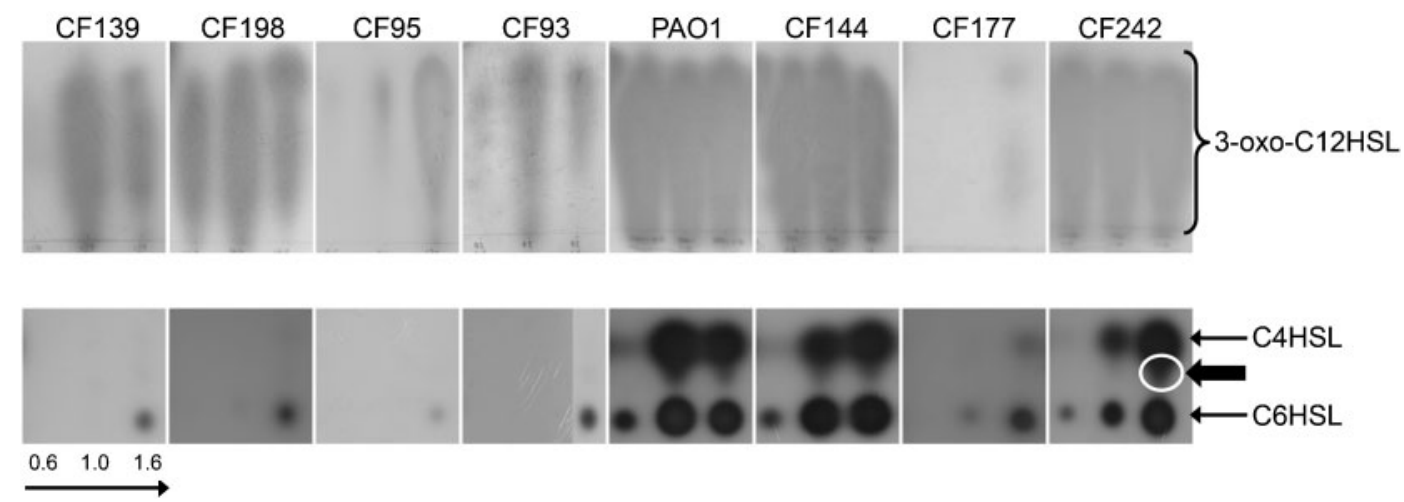

Fig. 1. Production of HSLs is strain dependent. Strains were grown to mid-exponential phase $\left(A_{600} 0.6\right)$, late-exponential phase $\left(A_{600} 1.0\right)$ and stationary phase $\left(A_{600} 1.6\right)$ and HSLs were extracted twice and detected using TLC as described in Methods. For each image, three lanes represent three growth phases $\left(A_{600} 0.6,1.0,1.6\right)$ in that order, as shown for strain CF139. The top panel shows long-chain HSLs and the bottom panel short-chain HSLs. The arrow and circle indicate an extra uncharacterized HSL. 
both long- and short-chain HSLs (Fig. 1) and this is consistent with adaptation of these strains to the host.

\section{Cell-free supernatant from $P$. aeruginosa can influence $C$. albicans morphology in an HSL- dependent manner}

Pure HSLs and structurally similar molecules can prevent the yeast to hyphal switch in C. albicans but it was not known whether this effect could also occur with complex bacterial secretions from clinical strains. The variation in levels of HSLs is also likely to impact on how different strains of $P$. aeruginosa interact with both the host and with other micro-organisms such as $C$. albicans that may be present in the lung. In support of this, we found that supernatants from some strains, when added to actively growing cultures of $C$. albicans, inhibited the yeast to hyphal switch in an HSL-dependent manner (Fig. 2). Supernatants from the high HSL producer, CF144, and the low HSL producer, CF177, were added to Candida cultures as they were induced to switch from the yeast to the hyphal form. We also included supernatant from the characterized strain $P$. aeruginosa PAO1, which produces high levels of HSLs (Fig. 1). We found that strains that produced high amounts of HSLs (PAO1 and CF144) inhibited the yeast to hyphal switch (Fig. 2c, d), whereas the low/non-producing strain (CF177) was unable to influence the transition (Fig. 2a, b). These data suggest that this is an
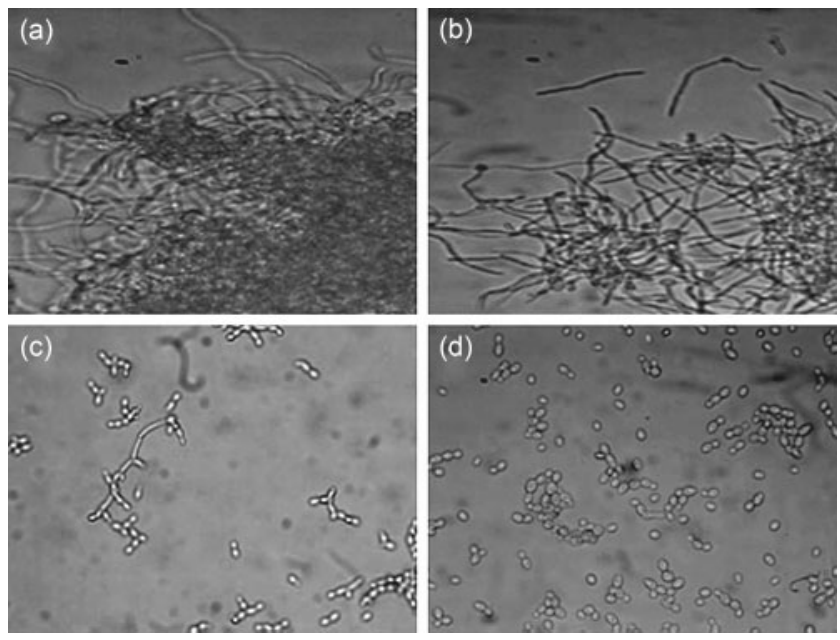

Fig. 2. Cell-free supernatant from $P$. aeruginosa influences $C$. albicans morphology. Supernatants from $P$. aeruginosa strains were sterilized by filtration and concentrated $\times 20$ by lyophilization and resuspension in purified water. Sterile, concentrated supernatants, at a final concentration of $\times 2$, were then added to $C$. albicans yeast cells that were induced to grow in filament form. Supernatants from strains PAO1 and CF144 inhibited the yeast to hyphal switch (c, d), whereas supernatant from strain CF177 (b) did not. (a) C. albicans hyphal control, (b) C. albicans +CF177 supernatant, (c) C. albicans +PAO1 supernatant and (d) C. albicans + CF144 supernatant. Magnification $\times 400$.
HSL-dependent phenomenon. To rule out the possibility that other molecules in the supernatant may prevent CF177 from mediating the effect, we performed an add-back experiment in which pure C12HSL was added to CF177 supernatant. This supplemented supernatant now inhibited the yeast to hyphal transition (data not shown). Further support for the role for long-chain HSLs came from experiments that showed that a $\mathrm{HSL}^{-}$mutant of PAO1,

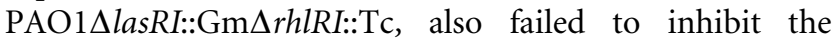
morphological switch (data not shown). Similar effects were reported previously with pure HSLs but the mechanism by which this effect is mediated is not known (Hogan \& Kolter, 2002; Hogan et al., 2004). The morphological switch in C. albicans involves the activity of two distinct signal transduction systems, a RAS-cAMPprotein kinase A pathway and a mitogen-activated protein kinase pathway, and it is believed that farnesol modulates one or both of these pathways in some way (Biswas et al., 2007; Enjalbert \& Whiteway, 2005; Hornby et al., 2001; Liu, 2001; Sato et al., 2004; Whiteway, 2000). As farnesol is also a 12-carbon molecule, it is tempting to speculate that 3oxo-C12HSL recognizes the same target as the C. albicans auto-signal, but this remains to be determined.

\section{Farnesol reduces swarming motility while having no effect on adhesion or growth in $P$. aeruginosa}

We were also interested in the effects of the Candida secreted signal, the 12-carbon molecule farnesol, on the clinical strains of $P$. aeruginosa. We therefore examined the effects that addition of farnesol had on three $P$. aeruginosa traits that have been reported as being important for virulence: swarming motility, growth and biofilm formation. As biofilm formation is influenced by HSLs and PQS, and the addition of farnesol reduces PQS levels, we investigated the ability of each strain to adhere to polyvinyl chloride (PVC) plates in the presence and absence of farnesol. Despite the phenotypic differences in the strains (growth rates, HSL production and colony morphology), no significant difference in the ability of the bacteria to adhere to PVC was detected among the strains and addition of $300 \mu \mathrm{M}$ farnesol had no effect on this adherence (data not shown). We also observed no effect on the growth of $P$. aeruginosa in the presence of up to $300 \mu \mathrm{M}$ farnesol (data not shown). Differences between strains and effects of farnesol were observed, however, when swarming motility was assessed (Fig. 3). The high HSL-producing strains (PAO1, CF144 and CF242) were strong swarmers, the low producer CF95 a moderate swarmer, and the low producers CF139, CF198, CF93 and CF177 were unable to swarm (Fig. 3a, arrows mark the edge of the swarming colony). The ability of strains PAO1, CF144, CF242 and CF95 to swarm was almost completely abolished by the addition of $100 \mu \mathrm{M}$ farnesol. The effect of farnesol may be mediated via rhamnolipids as rhamnolipids are known to be required for swarming and are partregulated by $\mathrm{PQS}$, which is known to be downregulated by farnesol. The reduction in PQS, pyocyanin production and 


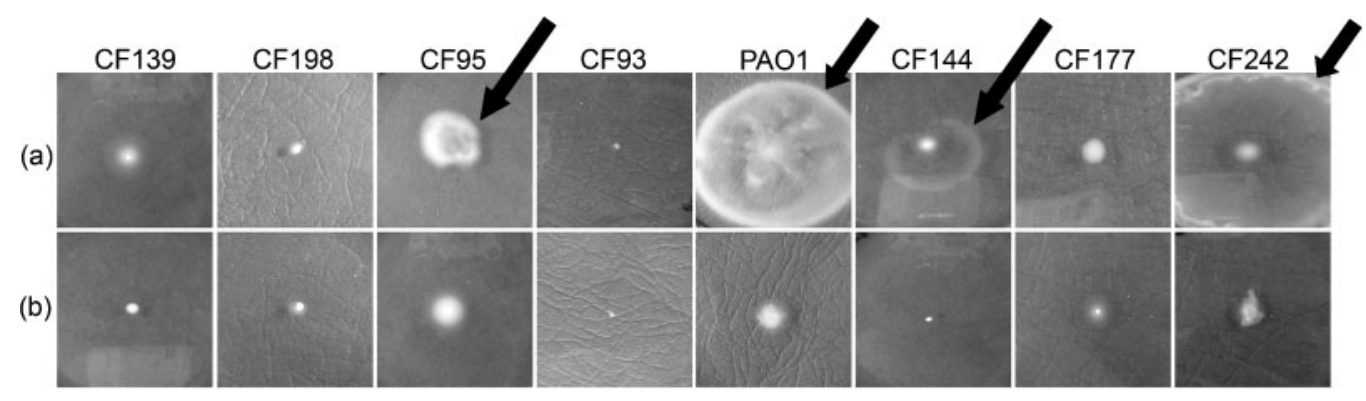

Fig. 3. Farnesol inhibits swarming in $P$. aeruginosa. (a) Swarming motility of $P$. aeruginosa on $0.5 \%$ Eiken agar plates. (b) Media were supplemented with $100 \mu \mathrm{M}$ farnesol and swarming was almost abolished. Swarm edges are indicated by arrows.

swarming motility may also have implications for the interaction between $P$. aeruginosa and the host.

\section{Importance of interspecies signalling in disease}

From the data above and in the literature, it is clear that the in vivo interaction between $P$. aeruginosa and C. albicans is likely to be complex and that it is possible to predict some consequences of these interactions. During the acute phase of infection when a strain of $P$. aeruginosa is secreting high levels of HSLs, any Candida cells in that niche will remain in the yeast form. In contrast, during a chronic infection when HSL concentrations are likely to drop, C. albicans may be able to trigger a switch to filamentous growth, possibly leading to formation of a fungal biofilm with the potential for invasive growth. Relatively little is known about the importance of fungal biofilms in disease, but this is an emerging topic of research. The possible cytotoxic effects of some strains of $P$. aeruginosa are an additional consideration as it has been shown that some strains of $P$. aeruginosa destroy Candida hyphae (but not yeast cells) (Hogan \& Kolter, 2002). In contrast, fungal signals can affect Pseudomonas traits important for gene regulation and motility, and are likely to impact on the ultrastructure of the bacterial biofilm.

In conclusion, it is emerging that the structure of the microbial community in the lung may have significant consequences for the nature of disease in CF patients. The key challenges now are to determine mechanistically precise details of how Pseudomonas and Candida interact, and to devise methods to study this interaction in vivo. The ultimate aim will be to use this knowledge to manipulate, modify, or disrupt microbial ecology in the CF lung to the benefit of the patient. These data also have implications for interactions between bacteria and fungi that may occur in other clinical settings, for example the burn wound.

\section{ACKNOWLEDGEMENTS}

We thank J. S. Mattick for generous provision of the $\mathrm{HSL}^{-}$mutant, Pat Higgins for excellent technical support, and Lucy Holcombe for critical reading of the manuscript. We also thank F. Boyd and colleagues in UCC for helpful discussions over the course of this research. Work in F. O'Gara's and J. Morrissey's laboratories is supported by grants awarded by the European Union (05/EDIV/ FP107; MTKD-CT-2006-042062; O36314), the Higher Education Authority of Ireland (PRTI3), Science Foundation of Ireland (SFI 02/ IN.1/B1261; 04/BR/B0597), the Irish Environmental Protection Agency (EPA 2006-PhD-S-21), Enterprise Ireland (SC/02/517) and the Department of Agriculture (06RDC459; 06RDC506; RSF06321; RSF06377). G. McAlester thanks the Society for General Microbiology for funding support.

\section{REFERENCES}

Adams, C., Morris-Quinn, M., McConnell, F., West, J., Lucey, B., Shortt, C., Cryan, B., Watson, J. B. \& O'Gara, F. (1998). Epidemiology and clinical impact of Pseudomonas aeruginosa infection in cystic fibrosis using AP-PCR fingerprinting. J Infect 37, 151-158.

Bakare, N., Rickerts, V., Bargon, J. \& Just-Nubling, G. (2003). Prevalence of Aspergillus fumigatus and other fungal species in the sputum of adult patients with cystic fibrosis. Mycoses 46, 19-23.

Bauernfeind, A., Bertele, R. M., Harms, K., Horl, G., Jungwirth, R., Petermuller, C., Przyklenk, B. \& Weisslein-Pfister, C. (1987). Qualitative and quantitative microbiological analysis of sputa of 102 patients with cystic fibrosis. Infection 15, 270-277.

Baysse, C., Cullinane, M., Denervaud, V., Burrowes, E., Dow, J. M., Morrissey, J. P., Tam, L., Trevors, J. T. \& O'Gara, F. (2005). Modulation of quorum sensing in Pseudomonas aeruginosa through alteration of membrane properties. Microbiology 151, 2529-2542.

Beatson, S. A., Whitchurch, C. B., Semmler, A. B. \& Mattick, J. S. (2002). Quorum sensing is not required for twitching motility in Pseudomonas aeruginosa. J Bacteriol 184, 3598-3604.

Biswas, S., Van Dijck, P. \& Datta, A. (2007). Environmental sensing and signal transduction pathways regulating morphopathogenic determinants of Candida albicans. Microbiol Mol Biol Rev 71, 348-376.

Burns, J. L., Van Dalfsen, J. M., Shawar, R. M., Otto, K. L., Garber, R. L., Quan, J. M., Montgomery, A. B., Albers, G. M., Ramsey, B. W. \& Smith, A. L. (1999). Effect of chronic intermittent administration of inhaled tobramycin on respiratory microbial flora in patients with cystic fibrosis. J Infect Dis 179, 1190-1196.

Burrowes, E., Baysse, C., Adams, C. \& O'Gara, F. (2006). Influence of the regulatory protein RsmA on cellular functions in Pseudomonas aeruginosa $\mathrm{PAO} 1$, as revealed by transcriptome analysis. Microbiology 152, 405-418.

Calderone, R. A. \& Fonzi, W. A. (2001). Virulence factors of Candida albicans. Trends Microbiol 9, 327-335. 
Calfee, M. W., Shelton, J. G., McCubrey, J. A. \& Pesci, E. C. (2005). Solubility and bioactivity of the Pseudomonas quinolone signal are increased by a Pseudomonas aeruginosa-produced surfactant. Infect Immun 73, 878-882.

Cugini, C., Calfee, M. W., Farrow, J. M., III, Morales, D. K., Pesci, E. C. \& Hogan, D. A. (2007). Farnesol, a common sesquiterpene, inhibits PQS production in Pseudomonas aeruginosa. Mol Microbiol 65, 896-906.

Diggle, S. P., Winzer, K., Chhabra, S. R., Worrall, K. E., Camara, M. \& Williams, P. (2003). The Pseudomonas aeruginosa quinolone signal molecule overcomes the cell density-dependency of the quorum sensing hierarchy, regulates rhl-dependent genes at the onset of stationary phase and can be produced in the absence of LasR. Mol Microbiol 50, 29-43.

Diggle, S. P., Cornelis, P., Williams, P. \& Camara, M. (2006). 4Quinolone signalling in Pseudomonas aeruginosa: old molecules, new perspectives. Int J Med Microbiol 296, 83-91.

Diggle, S. P., Matthijs, S., Wright, V. J., Fletcher, M. P., Chhabra, S. R., Lamont, I. L., Kong, X., Hider, R. C., Cornelis, P. \& other authors (2007). The Pseudomonas aeruginosa 4-quinolone signal molecules HHQ and PQS play multifunctional roles in quorum sensing and iron entrapment. Chem Biol 14, 87-96.

Drenkard, E. \& Ausubel, F. M. (2002). Pseudomonas biofilm formation and antibiotic resistance are linked to phenotypic variation. Nature 416, 740-743.

Enjalbert, B. \& Whiteway, M. (2005). Release from quorum-sensing molecules triggers hyphal formation during Candida albicans resumption of growth. Eukaryot Cell 4, 1203-1210.

Finnan, S., Morrissey, J. P., O'Gara, F. \& Boyd, E. F. (2004). Genome diversity of Pseudomonas aeruginosa isolates from cystic fibrosis patients and the hospital environment. J Clin Microbiol 42, 5783-5792.

Giamarellou, H. (2000). Therapeutic guidelines for Pseudomonas aeruginosa infections. Int J Antimicrob Agents 16, 103-106.

Gillum, A. M., Tsay, E. Y. \& Kirsch, D. R. (1984). Isolation of the Candida albicans gene for orotidine-5' -phosphate decarboxylase by complementation of S. cerevisiae ura3 and E. coli pyrF mutations. Mol Gen Genet 198, 179-182.

Govan, J. R. \& Deretic, V. (1996). Microbial pathogenesis in cystic fibrosis: mucoid Pseudomonas aeruginosa and Burkholderia cepacia. Microbiol Rev 60, 539-574.

Gow, N. A. (1997). Germ tube growth of Candida albicans. Curr Top Med Mycol 8, 43-55.

Gow, N. A., Brown, A. J. \& Odds, F. C. (2002). Fungal morphogenesis and host invasion. Curr Opin Microbiol 5, 366-371.

Gupta, N., Haque, A., Mukhopadhyay, G., Narayan, R. P. \& Prasad, R. (2005). Interactions between bacteria and Candida in the burn wound. Burns 31, 375-378.

Heurlier, K., Denervaud, V. \& Haas, D. (2006). Impact of quorum sensing on fitness of Pseudomonas aeruginosa. Int J Med Microbiol 296, 93-102.

Hogan, D. A. (2006a). Quorum sensing: alcohols in a social situation. Curr Biol 16, R457-R458.

Hogan, D. A. (2006b). Talking to themselves: autoregulation and quorum sensing in fungi. Eukaryot Cell 5, 613-619.

Hogan, D. A. \& Kolter, R. (2002). Pseudomonas-Candida interactions: an ecological role for virulence factors. Science 296, 2229-2232.

Hogan, D. A., Vik, A. \& Kolter, R. (2004). A Pseudomonas aeruginosa quorum-sensing molecule influences Candida albicans morphology. Mol Microbiol 54, 1212-1223.

Holloway, B. W. \& Morgan, A. F. (1986). Genome organization in Pseudomonas. Annu Rev Microbiol 40, 79-105.
Hornby, J. M., Jensen, E. C., Lisec, A. D., Tasto, J. J., Jahnke, B., Shoemaker, R., Dussault, P. \& Nickerson, K. W. (2001). Quorum sensing in the dimorphic fungus Candida albicans is mediated by farnesol. Appl Environ Microbiol 67, 2982-2992.

Hube, B. (2006). Infection-associated genes of Candida albicans. Future Microbiol 1, 209-218.

Hughes, W. T. \& Kim, H. K. (1973). Mycoflora in cystic fibrosis: some ecologic aspects of Pseudomonas aeruginosa and Candida albicans. Mycopathol Mycol Appl 50, 261-269.

Kaleli, I., Cevahir, N., Demir, M., Yildirim, U. \& Sahin, R. (2007). Anticandidal activity of Pseudomonas aeruginosa strains isolated from clinical specimens. Mycoses 50, 74-78.

Kerr, J. (1994a). Inhibition of fungal growth by Pseudomonas aeruginosa and Pseudomonas cepacia isolated from patients with cystic fibrosis. J Infect 28, 305-310.

Kerr, J. R. (1994b). Suppression of fungal growth exhibited by Pseudomonas aeruginosa. J Clin Microbiol 32, 525-527.

Liu, H. (2001). Transcriptional control of dimorphism in Candida albicans. Curr Opin Microbiol 4, 728-735.

Liu, H. (2002). Co-regulation of pathogenesis with dimorphism and phenotypic switching in Candida albicans, a commensal and a pathogen. Int J Med Microbiol 292, 299-311.

Martin, C., Ichou, M. A., Massicot, P., Goudeau, A. \& Quentin, R. (1995). Genetic diversity of Pseudomonas aeruginosa strains isolated from patients with cystic fibrosis revealed by restriction fragment length polymorphism of the rRNA gene region. J Clin Microbiol 33, 1461-1466.

McGrath, S., Wade, D. S. \& Pesci, E. C. (2004). Dueling quorum sensing systems in Pseudomonas aeruginosa control the production of the Pseudomonas quinolone signal (PQS). FEMS Microbiol Lett 230, 27-34.

Mitchell, A. P. (1998). Dimorphism and virulence in Candida albicans. Curr Opin Microbiol 1, 687-692.

Naglik, J., Albrecht, A., Bader, O. \& Hube, B. (2004). Candida albicans proteinases and host/pathogen interactions. Cell Microbiol 6, 915-926.

Navarathna, D. H., Hornby, J. M., Krishnan, N., Parkhurst, A., Duhamel, G. E. \& Nickerson, K. W. (2007a). Effect of farnesol on a mouse model of systemic candidiasis, determined by use of a DPP3 knockout mutant of Candida albicans. Infect Immun 75, 1609-1618.

Navarathna, D. H., Nickerson, K. W., Duhamel, G. E., Jerrels, T. R. \& Petro, T. M. (2007b). Exogenous farnesol interferes with the normal progression of cytokine expression during candidiasis in a mouse model. Infect Immun 75, 4006-4011.

Nguyen, D. \& Singh, P. K. (2006). Evolving stealth: genetic adaptation of Pseudomonas aeruginosa during cystic fibrosis infections. Proc Natl Acad Sci U S A 103, 8305-8306.

Nickerson, K. W., Atkin, A. L. \& Hornby, J. M. (2006). Quorum sensing in dimorphic fungi: farnesol and beyond. Appl Environ Microbiol 72, 3805-3813.

Oliver, A., Canton, R., Campo, P., Baquero, F. \& Blazquez, J. (2000). High frequency of hypermutable Pseudomonas aeruginosa in cystic fibrosis lung infection. Science 288, 1251-1254.

Pfaller, M. A. \& Diekema, D. J. (2007). Epidemiology of invasive candidiasis: a persistent public health problem. Clin Microbiol Rev 20, 133-163.

Rahme, L. G., Stevens, E. J., Wolfort, S. F., Shao, J., Tompkins, R. G. \& Ausubel, F. M. (1995). Common virulence factors for bacterial pathogenicity in plants and animals. Science 268, 1899-1902.

Rahme, L. G., Ausubel, F. M., Cao, H., Drenkard, E., Goumnerov, B. C., Lau, G. W., Mahajan-Miklos, S., Plotnikova, J., Tan, M. W. \& other authors (2000). Plants and animals share functionally common 
bacterial virulence factors. Proc Natl Acad Sci U $S$ A 97, 8815-8821.

Ravn, L., Christensen, A. B., Molin, S., Givskov, M. \& Gram, L. (2001). Methods for detecting acylated homoserine lactones produced by Gram-negative bacteria and their application in studies of AHLproduction kinetics. J Microbiol Methods 44, 239-251.

Sato, T., Watanabe, T., Mikami, T. \& Matsumoto, T. (2004). Farnesol, a morphogenetic autoregulatory substance in the dimorphic fungus Candida albicans, inhibits hyphae growth through suppression of a mitogen-activated protein kinase cascade. Biol Pharm Bull 27, 751-752.

Schuster, M. \& Greenberg, E. P. (2006). A network of networks: quorum-sensing gene regulation in Pseudomonas aeruginosa. Int $J$ Med Microbiol 296, 73-81.

Shaw, P. D., Ping, G., Daly, S. L., Cha, C., Cronan, J. E., Jr, Rinehart, K. L. \& Farrand, S. K. (1997). Detecting and characterizing $\mathrm{N}$-acylhomoserine lactone signal molecules by thin-layer chromatography. Proc Natl Acad Sci U S A 94, 6036-6041.

Shiner, E. K., Rumbaugh, K. P. \& Williams, S. C. (2005). Interkingdom signaling: deciphering the language of acyl homoserine lactones. FEMS Microbiol Rev 29, 935-947.

Smith, R. S. \& Iglewski, B. H. (2003). P. aeruginosa quorum-sensing systems and virulence. Curr Opin Microbiol 6, 56-60.

Smith, J. J., Travis, S. M., Greenberg, E. P. \& Welsh, M. J. (1996). Cystic fibrosis airway epithelia fail to kill bacteria because of abnormal airway surface fluid. Cell 85, 229-236.

Smith, E. E., Buckley, D. G., Wu, Z., Saenphimmachak, C., Hoffman, L. R., D’Argenio, D. A., Miller, S. I., Ramsey, B. W., Speert, D. P. \& other authors (2006). Genetic adaptation by Pseudomonas aeruginosa to the airways of cystic fibrosis patients. Proc Natl Acad Sci U S A 103, 8487-8492.

Tan, M. W. \& Ausubel, F. M. (2000). Caenorhabditis elegans: a model genetic host to study Pseudomonas aeruginosa pathogenesis. Curr Opin Microbiol 3, 29-34.

Wade, D. S., Calfee, M. W., Rocha, E. R., Ling, E. A., Engstrom, E., Coleman, J. P. \& Pesci, E. C. (2005). Regulation of Pseudomonas quinolone signal synthesis in Pseudomonas aeruginosa. J Bacteriol 187, 4372-4380.

Whitchurch, C. B., Beatson, S. A., Comolli, J. C., Jakobsen, T., Sargent, J. L., Bertrand, J. J., West, J., Klausen, M., Waite, L. L. \& other authors (2005). Pseudomonas aeruginosa fimL regulates multiple virulence functions by intersecting with Vfr-modulated pathways. Mol Microbiol 55, 1357-1378.

Whiteway, M. (2000). Transcriptional control of cell type and morphogenesis in Candida albicans. Curr Opin Microbiol 3, 582-588.

Whiteway, M. \& Oberholzer, U. (2004). Candida morphogenesis and host-pathogen interactions. Curr Opin Microbiol 7, 350-357.

Williams, P. (2002). Quorum sensing: an emerging target for antibacterial chemotherapy? Expert Opin Ther Targets 6, 257-274.

Wolfgang, M. C., Kulasekara, B. R., Liang, X., Boyd, D., Wu, K., Yang, Q., Miyada, C. G. \& Lory, S. (2003). Conservation of genome content and virulence determinants among clinical and environmental isolates of Pseudomonas aeruginosa. Proc Natl Acad Sci U S A 100, 8484-8489. 